\title{
Investigation of the Plasma-Electrodes Interfaces by Their Participation in the Simulation Region
}

\author{
M. Gharaeinia, Sh. Saviz* And A.H. Sari \\ Plasma Physics Research Center, Science and Research Branch, \\ Islamic Azad University, Tehran, Iran
}

Received: 03.11.2019 \& Accepted: 14.05.2020

Doi: 10.12693/APhysPolA.138.392

*e-mail: Shahrooz.Saviz@srbiau.ac.ir

\begin{abstract}
A plasma torch has many applications and the understanding of the electrical and thermal phenomena that occur inside it is necessary to control its stability and extend its lifetime. Mumerical simulation can provide a powerful tool, however, the good simulation should be close to reality, and unrealistic boundary conditions should not be used in the modeling. In the most of performed studies so far, the simulation region contains only the space between the electrodes (plasma), and the unrealistic boundary conditions have been applied at the electrode surface. Taking such boundary conditions might produced inaccurate results. Certainly, the thermal and electrical properties are not only dependent on the plasma medium but also on the electrodes. In this study, in order to eliminate such boundary conditions, in addition to the plasma passage area, both electrodes are included in the simulation region. Three-dimensional, time-dependent and non-equilibrium simulation is performed and the plasma temperature distribution is predicted. The electric current path along the cathode-plasma-anode is determined without applying any unrealistic boundary conditions. In fact, the obtained result is in good agreement with the experiment. The electric current passes through the tapered section of the anode and this finding is well consistent with that of the eroded experimental sample. The heat transfer coefficient distribution at the anode surface is also obtained. The maximum heat transfer coefficient is about $1.6 \times 10^{4} \mathrm{~W} /\left(\mathrm{m}^{2} \mathrm{~K}\right)$ at the location of the arc attachment. A comparison with some conventional unrealistic boundary conditions indicates that some of them are inaccurate and need to be reviewed.
\end{abstract}

topics: plasma torch, numerical simulations, boundary conditions

\section{Introduction}

A plasma torch is widely used in many applications such as plasma spray coating, chemical and powder synthesis, extractive metallurgy, toxic waste treatment and pyrolysis [1]. A conventional non-transferred DC plasma torch typically consists of two electrodes and the space between them. The gas passes through the space between the electrodes and the plasma is formed by applying an electric potential difference. The high current density at the location of the arc attachment causes that the electrodes become eroded [2]. By predicting the electrical and thermal properties of the interface between the electrodes and the plasma, it is possible to extend the lifetime of the plasma torch and optimize its plasma. Since the experimental results are limited because of the expensive equipment and the difficulty of extracting the results from within the plasma torch, one can use simulations which are very cost-effective [3].

Many simulations have been carried out which have greatly influenced the prediction and optimization of the plasma torch. In most of the works the computational domain has only included the space between the electrodes (plasma).
However, inserting the electrodes into the simulation area can be very interesting. This leads to a continuity in the electrical and magnetic properties of the plasma-electrode interfaces. This also allows to avoid the need to apply unrealistic boundary conditions for heat transfer and electric current at the electrode surface and enables more reliable results. For example, in most literature, a symmetric Gaussian current density distribution is applied as the boundary condition at the cathode tip while in reality this cannot be the maximum current density, exactly at the center of the cathode tip [4]. The accurate modeling of heat transfer is also important in a simulation of a non-transferred DC plasma torch because a high heat flux at the location of the arc attachment causes anode erosion and shortens its lifetime [5].

Alaya et al. [6] incorporated the cathode into the simulation area. Using the local thermodynamic equilibrium (LTE) model and applying electric current density on the rear face of the cathode, they calculated the heat transfer and current density distributions at the cathode tip surface. However, no model has yet been presented in which both electrodes have been simulated. Incorporating the anode into the simulation greatly 
increases the complexity of the model and the cost of computation [7]. For example, it must be a nonequilibrium model (NLTE). One of the limitations of the LTE model is the need to apply unrealistic boundary conditions for the passage of electric current at the plasma-anode interface. Due to water cooling, the temperature of the gas near the anode surface drops below $1000 \mathrm{~K}$ and the electrical conductivity of the gas is not enough to allow electric charges to pass through the arc column to the electrodes. It is also accepted that the results of NLTE models are more realistic and closer to experiments [8]. Therefore, it is better to perform the simulation with a non-equilibrium model and insert electrodes into the simulation area.

In this study, a three-dimensional, nonequilibrium and time-dependent plasma torch model is simulated and in addition to the cathode, the anode has also been incorporated into the simulation region. A method similar to that presented in [6] is used to apply the electric current to the cathode. No unrealistic model is used to determine the location of the arc attachment on the surface of the electrodes.

This model has been used to:

- predict more eroded areas by determining the distribution of the electric current density at the anode surface,

- determine the surface temperature of electrodes, predict heat transfer distribution at the anode surface and make a comparison with the results of applying unreal boundary conditions, where the electrodes were not part of the simulated region.

\section{Model description}

\subsection{Assumptions}

The model is considered based on the following main assumptions:

- the plasma is in non-equilibrium conditions and the temperature of the electrons and heavy particles are different,

- the plasma is considered optically thin,

- the gravitational effects are negligible,

- the path of the electric current passing through the cathode-plasma-anode is determined by solving the governing equations in the three zones,

- no models are used for arc reattachment.

\subsection{Governing equations}

Plasma behaves like a fluid containing electrons and argon particles, which include ground-state atoms (Ar), electronically excited atoms $\left(\mathrm{Ar}^{*}\right)$, atomic ions $\left(\mathrm{Ar}^{+}\right)$and molecular ions $\left(\mathrm{Ar}_{2}^{+}\right)$[9].
Processes taken into account.

TABLE I

\begin{tabular}{c|l}
\hline \hline Process & \multicolumn{1}{c}{ Reaction } \\
\hline 1 & $\mathrm{M}+\mathrm{e} \rightarrow \mathrm{M}+\mathrm{e}\left(\mathrm{M}=\mathrm{Ar}, \mathrm{Ar}^{+}\right)$ \\
2 & $\mathrm{Ar}+\mathrm{e} \rightarrow \mathrm{Ar}^{*}+\mathrm{e}$ \\
3 & $\mathrm{Ar}^{*}+\mathrm{e} \rightarrow \mathrm{Ar}+\mathrm{e}$ \\
4 & $\mathrm{Ar}+\mathrm{e} \rightarrow \mathrm{Ar}^{+}+2 \mathrm{e}$ \\
5 & $\mathrm{Ar}^{*}+\mathrm{e} \rightarrow \mathrm{Ar}^{+}+2 \mathrm{e}$ \\
6 & $2 \mathrm{Ar}^{*} \rightarrow \mathrm{Ar}+\mathrm{Ar}^{+}+\mathrm{e}$ \\
7 & $\mathrm{Ar}^{\mathrm{Ar}} \rightarrow 2 \mathrm{Ar}$ \\
8 & $\mathrm{Ar}^{+}+2 \mathrm{e} \rightarrow \mathrm{Ar}+\mathrm{e}$ \\
9 & $\mathrm{Ar}^{*} \mathrm{Ar}^{+}+\mathrm{e} \rightarrow 2 \mathrm{Ar}$ \\
10 & $\mathrm{Ar}_{2}^{+}+\mathrm{e} \rightarrow \mathrm{Ar}+\mathrm{Ar}^{+}+\mathrm{e}$
\end{tabular}

The processes considered in the model are summarized in Table I. These reactions include the elastic processes, scattering, excitation, deexcitation, etc. The reactions comprise electron-induced reactions which depend on the temperature of the electrons, and the reactions of heavy species which are dependent on the temperature of the gas. The reaction rates have been taken from [9] and the software database [10].

In description one uses the mass conservation equation

$$
\frac{\partial \rho}{\partial t}+\nabla \cdot(\rho \boldsymbol{v})=0 .
$$

where the total mass density $\rho$ moves with particle velocity $\boldsymbol{v}=(u, \nu, w)$. The Navier-Stokes equations have the following form

$\frac{\partial(\rho u)}{\partial t}+\nabla \cdot(\rho \boldsymbol{v} u)=-\frac{\partial p}{\partial x}+\nabla \cdot(\mu \boldsymbol{v} u)+\left(\boldsymbol{j}_{e} \times \boldsymbol{B}\right)_{x}$,
$\frac{\partial(\rho \nu)}{\partial t}+\nabla \cdot(\rho \boldsymbol{v} \nu)=-\frac{\partial p}{\partial y}+\nabla \cdot(\mu \boldsymbol{v} \nu)+\left(\boldsymbol{j}_{e} \times \boldsymbol{B}\right)_{y}$,
$\left.\frac{\partial(\rho w)}{\partial t}+\nabla \cdot(\rho \boldsymbol{v} w)=-\frac{\partial p}{\partial z}+\nabla \cdot(\mu \boldsymbol{v} w)+\boldsymbol{J}_{e} \times \boldsymbol{B}\right)_{z}$,

where $p$ is the pressure, $\boldsymbol{J}_{e}$ is the electron electric current density vector. The viscosity $\mu$ is determined from the mix kinetic theory of gases. The conservation equations for heavy particle species $\alpha$ are written as

$$
\frac{\partial\left(\rho Y_{\alpha}\right)}{\partial t}+\nabla\left(\rho \boldsymbol{v} Y_{\alpha}\right)=\nabla \cdot \boldsymbol{J}_{e}+S_{\alpha}
$$

with $Y_{\alpha}$ as a species mass fraction, and $S_{\alpha}$ as a production rate of the particle $\alpha$.

Note that the energy equations for electrons and heavy particles should be solved separately. The electron transport is given as

$$
\frac{\partial n_{e}}{\partial t}+\nabla \cdot \boldsymbol{\Gamma}_{e}=S
$$

where the electron density flux $\boldsymbol{\Gamma}_{e}$ is equal to

$$
\boldsymbol{\Gamma}_{e}=\mu_{e} n_{e} \nabla \varphi-D_{e} \nabla n_{e} .
$$

Here, $n_{e}$ denotes the density of electron number, $\mu_{e}$ is the electron mobility, $D_{e}$ is the electron diffusion coefficient, and $\varphi$ is the electron potential. 
To obtain the electron temperature $T_{e}$ one solves the following equation

$$
\begin{aligned}
& \frac{3}{2} \frac{\partial\left(n_{e} T_{e}\right)}{\partial t}+\nabla\left(\frac{5}{2} T_{e} \boldsymbol{\Gamma}_{e}-k_{e} \nabla T_{e}\right)= \\
& P-n_{e} \sum_{r} n_{r} k_{r} \epsilon_{r},
\end{aligned}
$$

where $k_{e}=\frac{5}{2} n_{e} D_{e}$ is the thermal conductivity of electron. The power density $P$ is the total energy absorbed by electrons, such as Joule heating, inductive heating and external heating, i.e.,

$$
P=P_{\text {Joule }}+P_{\text {ind }}+P_{\text {ext }} \text {. }
$$

The last term on the right-hand side of (6) accounts for the electron energy loss due to the electroninduced reactions.

Now, the energy equation for heavy particles reads as

$$
\begin{aligned}
& C_{p}\left[\frac{\partial(\rho T)}{\partial t}+\nabla(\rho \boldsymbol{v} T)\right]=\nabla \cdot\left(k_{\mathrm{B}} \nabla T\right) \\
& \quad+\sum_{i} J_{i} C_{p_{i}} \cdot \nabla T+\dot{Q}-\sum_{r} \epsilon_{r} \dot{\omega}_{r}+\sum_{l=\text { elastic }} \epsilon_{l} \dot{\omega}_{l} .
\end{aligned}
$$

where $C_{p}$ is the specific heat with constant pressure. Gas heating sources have been added to include such phenomena: electron elastic and inelastic collisions, ion ohmic heating $\dot{Q}$, ion-surface recombination and kinetic impact.

Maxwell's electromagnetic equations applied for the magnetic vector potential $\mathbf{A}$ are [2]

$$
\begin{aligned}
& \nabla \cdot \boldsymbol{J}=0, \\
& \boldsymbol{E}=-\nabla \varphi, \\
& \boldsymbol{J}=\sigma_{e}(-\nabla \varphi+\boldsymbol{v} \times \boldsymbol{B}) \\
& \nabla^{2} \boldsymbol{A}=-\mu_{0} \boldsymbol{J}, \\
& \boldsymbol{B}=\nabla \times \boldsymbol{A},
\end{aligned}
$$

In the case of turbulence the standard $K-\epsilon$ model applies. The effect of turbulence on heat transfer and mass diffusion is accomplished, mainly through expressions:

$$
\begin{aligned}
& k_{\mathrm{eff}}=k+\frac{\mu_{t} C_{p}}{\operatorname{Pr}_{t}} \\
& D_{\text {eff }}=D+\frac{\mu_{t}}{S c_{t}} .
\end{aligned}
$$

The turbulent Prandtl number is $\mathrm{Pr}_{t}$, while the turbulent component of viscosity is $\mu_{t}=\rho C_{\mu} \frac{K^{2}}{\epsilon}$. The model we are considering performs the characteristic description for the turbulent kinetic energy $K$ and dissipation rate of turbulent kinetic $\epsilon[11]$. Namely,

$$
\begin{aligned}
& \frac{\partial(\rho K)}{\partial t}+\nabla \cdot(\rho K \boldsymbol{v})= \\
& \nabla \cdot\left(\frac{\mu_{t}}{\sigma_{K}}(\nabla K)\right)+2 \mu_{t} \boldsymbol{E}_{i j}^{2}+\rho \epsilon, \\
& \frac{\partial(\rho \epsilon)}{\partial t}+\nabla \cdot(\rho \epsilon \boldsymbol{v})= \\
& \nabla \cdot\left(\frac{\mu_{t}}{\sigma_{\epsilon}}(\nabla \epsilon)\right)+2 \mu_{t} \boldsymbol{E}_{i j}^{2} \frac{\epsilon C_{1 \epsilon}}{K}-\rho \epsilon \frac{\epsilon C_{2 \epsilon}}{K} .
\end{aligned}
$$

The constant values in the $K-\epsilon$ model, which are calculated using data fitting for a wide range of turbulent fluids, are [2]

$$
\begin{aligned}
& C_{\mu}=0.09, \quad \sigma_{K}=1.00, \quad \sigma_{\epsilon}=1.30, \\
& C_{1 \epsilon}=1.44, \quad C_{2 \epsilon}=1.92 .
\end{aligned}
$$

The heat transfer within the electrodes, which includes Joule heating and thermal conductivity, is described as [9]:

$$
\rho_{S} C_{p S} \frac{\partial T}{\partial t}=\nabla \cdot\left(\lambda_{S} \nabla T\right)+\sigma_{S} \boldsymbol{E}^{2}
$$

In above expression $\rho_{S}$ is the solid mass density, $C_{p S}$ is the solid heat conductivity, $\sigma_{S}$ is the solid electrical conductivity, and $\lambda_{S}$ is the solid thermal conductivity.

\subsection{Model setup and boundary condition}

The geometry used in this study corresponds to the SG-100 plasma torch. The simulation region is shown in Fig. 1. This region consists of three volumes: anode, cathode and plasma motion space. The simulation region meshes to more than $2 \times 10^{5}$ cells. The gas enters from the inlet and after passing through the space between the electrodes, the plasma is formed and released from the outlet.

Table II shows the details of the intended boundary conditions. In the torch inlet, the gas temperature is $1000 \mathrm{~K}$ with the flow rate of $50 \mathrm{Slpm}$. At the outlet, a fixed pressure condition is applied.

The anode is made of copper and the cathode is of tungsten. The outer surface of the anode is isothermal to its surroundings and has a temperature of $300 \mathrm{~K}$. In the interface between the plasma and the electrodes, a no-slip condition is considered $(u=\nu=w=0)$ [12]. The electron temperature at the plasma-electrode interfaces is thermal flux balanced, in which the electron temperatures in the cells surrounding the electrodes face are used to set their electron temperature. At the interface between plasma and the electrodes, tangential components of the electric field and magnetic vector potential are zero [10]. The extrapolation boundary condition for the magnetic vector potential at the inlet and outlet specifies that each component (spatial, real/imaginary) will have no gradient in the direction of the face normal [10].

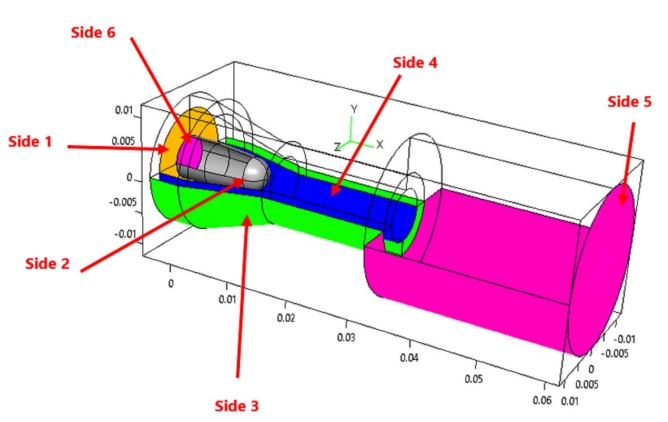

Fig. 1. Computational domain and boundary sides. 
Summary of boundary conditions.

TABLE II

\begin{tabular}{l|c|c|c|c}
\hline \multicolumn{1}{c|}{ Boundary } & Velocity & $T[\mathrm{~K}]$ & Magnetic & $T T_{e}[\mathrm{eV}]$ \\
\hline Side 1: inlet & $V_{\text {in }}$ & 1000 & extrapolation & 0.086 \\
Side 2: cathode & no-slip & predict & perfect conductor & thermal flux balanced \\
Side 3: anode outer surface & - & isothermal & perfect conductor \\
Side 4: anode-plasma interface & no-slip & predict & perfect conductor & thermal flux balanced \\
Side 5: outlet & fixed pressure & 300 & extrapolation & $\frac{\partial T_{e}}{\partial n}=0$ \\
Side 6: rear surface of the cathode & - & 500 & perfect conductor
\end{tabular}

The total electric current of the plasma is $600 \mathrm{~A}$. A constant current density is applied to the rear surface of the cathode, as it was done in [6], and the current flows through the cathode-plasmaanode regions. There are no additional constraints to determine the path of the electric current across different regions. Therefore, it is possible to obtain the current density distribution in the interface of electrodes and plasma. The arc ignition is initiated by an initial concentration of argon ions throughout the region between the electrodes.

\section{Results and discussion}

\subsection{Plasma characteristics}

In this study, in addition to the cathode, we also incorporated the anode into the computational domain to obtain more information about the plasma interface with electrodes. No unrealistic model has been used to determine the location of arc attachment and the passage of electric current through the interface between the electrodes and plasma.

Figure 2 shows gas temperature and velocity distributions inside the plasma torch. The temperature distribution is not symmetric and has a higher temperature at the point indicated by the blue positive sign. The highest plasma electron temperature is of the order of $37500 \mathrm{~K}$, which is comparable to the reported result in [13]. The predicted potential drop between the anode and the cathode is $28 \mathrm{~V}$. In comparison, the experimental drop is $29.5 \mathrm{~V}$ [13].

\subsection{Electric current}

The distribution of electric current density vectors is shown in Fig. 3. The high density of the electric current vectors is well observed in the arc crossing path. The electric current passage through the entire simulation region is obtained only by considering the total current of $600 \mathrm{~A}$ and applying it to the rear end of the cathode. No additional boundary conditions are applied for the passage of the current at the surfaces of the electrodes.

When we make a comparison with the plasma temperature distribution in Fig. 2, it is evident that the temperature is higher in areas with higher current density. It is clear that most of the current flow takes place through the tapered section of the anode.

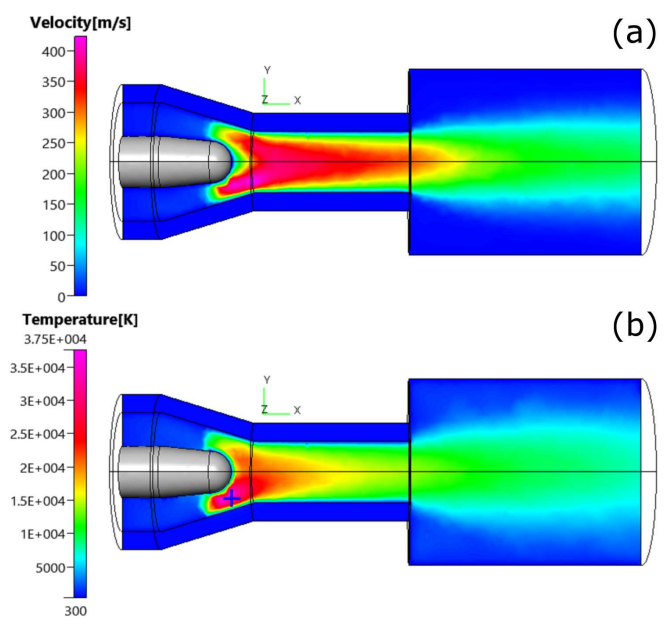

Fig. 2. Temperature and velocity distributions of plasma through the vertical cross-section.

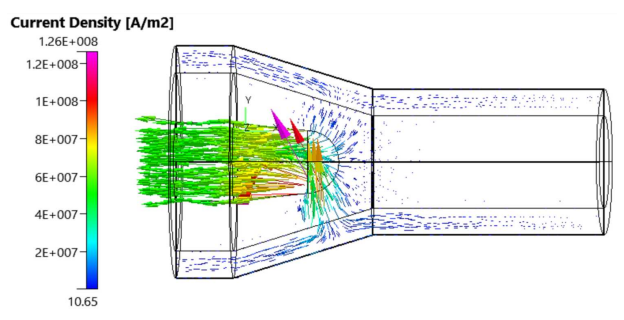

Fig. 3. Current density distribution (vectors color scale) through the vertical cross-section.

Figure 4 shows the overlap of electric current density distribution in this work, with an eroded anode sample provided in [13] (with permission from the Author). The electric current passage area fits well with the eroded zone. The electric current passes through the tapered section of the anode and this result is well consistent with that of the eroded experimental sample. According to the Steenbeck principle, when the arc current and working conditions keep constant, the arc is always located in a position with low arc voltage. When the arc attaches to the taper section of the anode, the arc voltage is lower than that in the columned section [14].

Importantly, due to the higher charge density at the sharp points of the metals, the rough area between the conical and the cylindrical part of 


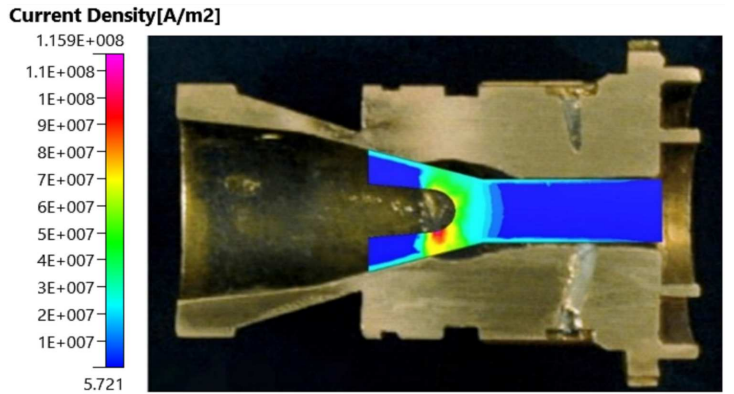

Fig. 4. Overlap of electric current density distribution with an eroded anode sample provided in [13] (with permission from the Author).

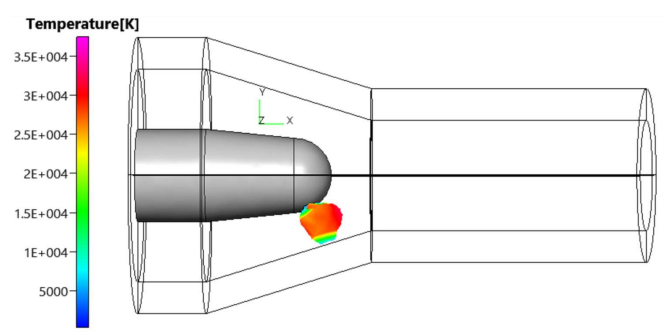

Fig. 5. The electron temperature isosurface of $27000 \mathrm{~K}$ and the temperature distribution over it.

the anode should be smoothed. The electron temperature isosurface of $27000 \mathrm{~K}$ (which resembles the shape of the arc) and the temperature distribution over it, is shown in Fig. 5. The temperature of the electrons is very high, while the plasma temperature varies at different points on the isosurface and decreases sharply near the electrodes. This is the advantage of using a non-equilibrium model, i.e., the temperature of the electrons and thus their conductivity is high enough for the electric current to be easily transmitted between the electrodes.

It is also possible that the most common boundary condition for the electric current on the cathode surface is the definition of the electric current density profile at its surface. Usually, in the literature, a symmetric profile is used for current density at the cathode surface [5]:

$$
J_{\text {Cath }}=J_{\text {Cath } 0} \exp \left(-\left(\frac{r}{R_{\text {Cath }}}\right)^{n_{\text {Cath }}}\right),
$$

where $J_{\text {Cath }}$ is the electric current density at the tip of the cathode, and $r$ is the radial coordinate measured from the torch axis, and the adjustable parameters $J_{\text {Cath } 0}, R_{\text {Cath }}$ and $n_{\text {Cath }}$ are determined by experimental measurements. In (20) it is assumed that the largest amount of the electric current passes through the center of the cathode tip. However, in reality, this cannot be the maximum current density, exactly at the center of the cathode tip [4]. Therefore, this is an unrealistic boundary condition. In this study, by inserting the cathode into the simulation area, the current density distribution at the tip of the cathode is also determined.

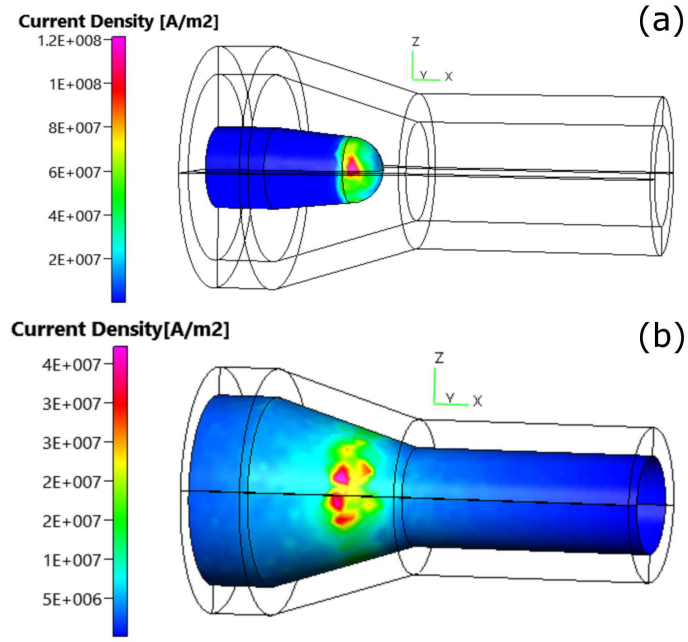

Fig. 6. The distribution of current density at the anode and cathode surface.

Thermal and magnetic coupling in the interface of electrodes and plasma makes it possible to predict the temperature and current density distributions at the surface of the electrodes. These distributions are shown in Fig. 6. The arc attaches to the points of the cathode tip which are closest to the anode surface. It should be noted that the electric current does not pass through a singular point, and the electric current density is higher in the arc direction. The predicted maximum current density at the cathode tip $1.2 \times 10^{8} \mathrm{~A} / \mathrm{m}^{2}$ is in good agreement with the experimental value $10^{8} \mathrm{~A} / \mathrm{m}^{2}$, and the value obtained by Alaya et al. [6].

The location of the arc-root attachment with the anode surface is shown in Fig. 6b. The difference of the electric potential between the electrodes causes the electric charges in the plasma to move toward the electrodes, thereby causing the electric current to pass between them. Although, most of the current passes through the arc, there is also the current passing elsewhere.

\subsection{Heat transfer}

In simulations where the cathode is not part of the simulation region, the surface temperature of the cathode is usually approximated by a Gaussian profile from $500 \mathrm{~K}$ at the inlet to $3600 \mathrm{~K}$ at the cathode tip. This boundary condition, of course, is not perfect since at the location of the arc attachment, the temperature will be higher.

Here, the thermal and magnetic coupling of the cathode and plasma allows for predicting the temperature distribution of the cathode surface. Figure 7 a shows the temperature distribution of the cathode surface. This distribution results from calculations with the only input data being the temperature value at the rear face of the cathode $(500 \mathrm{~K})$. The maximum temperature at the tip of the cathode is $3597 \mathrm{~K}$, which is in agreement with the unrealistic boundary condition applied in 


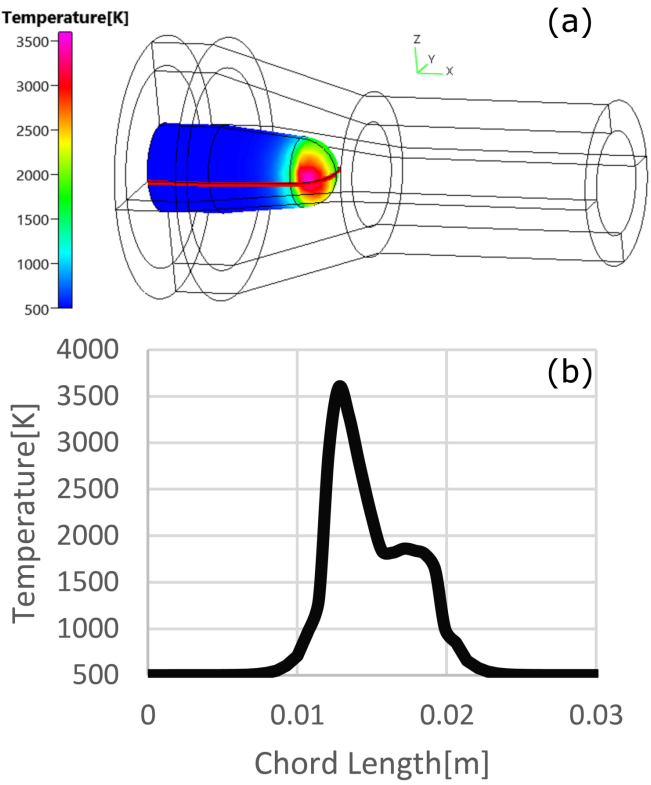

Fig. 7. Cathode surface: (a) Temperature distribution, (b) Temperature profile along the red chord.

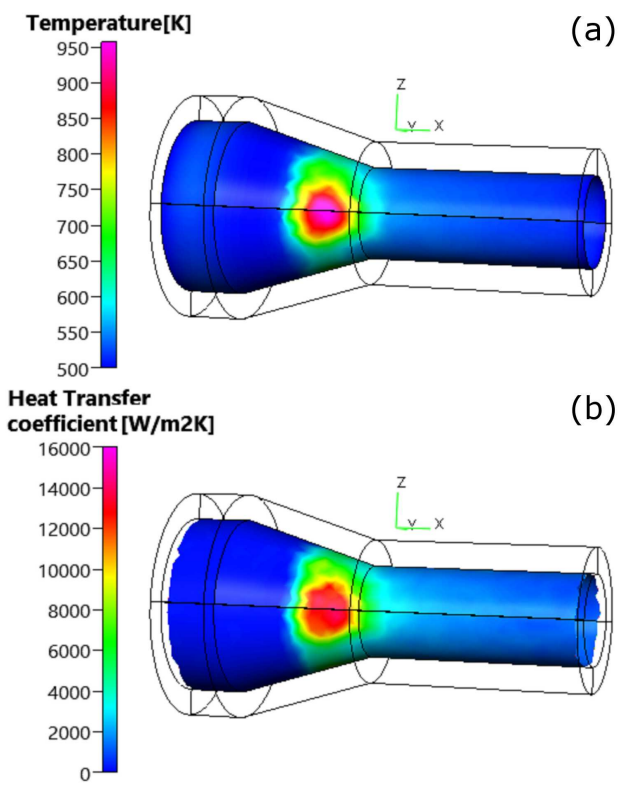

Fig. 8. Anode surface: (a) Temperature distribution, (b) Heat transfer coefficient distribution.

the literature. However, the maximum temperature is not exactly at the center of the cathode tip. The cathode temperature profile along a chord at the cathode surface (red chord in Fig. 7a) is shown in Fig. 7b. Obviously, this is not a normal Gaussian distribution.

It is very important to determine the surface temperature of the anode inside the plasma torch. Certainly, in places with higher temperatures, more erosion occurs. Figure 8a shows the temperature distribution of the anode surface. At the location of the arc attachment, the surface temperature of the anode is about $960 \mathrm{~K}$.
Usually, a simplified boundary condition for heat transfer is applied at the anode surface [8]:

$$
q_{\text {Wall }}=-\left.k\left(\frac{\partial T}{\partial n}\right)\right|_{\text {Wall }}=h_{W}\left(T-T_{W}\right) .
$$

The value of $h_{W}$ is usually considered to be in the order of $10^{5}$. For example, in $[13,15]$ this coefficient is considered to be $2 \times 10^{4} \mathrm{~W} /\left(\mathrm{m}^{2} \mathrm{~K}\right)$. But here by inserting the anode into the simulation area, it is no longer necessary to use this boundary condition and the surface temperature of the anode is automatically determined. As a result, the heat transfer coefficient can also be obtained depending on the surface temperature of the anode at different points. Figure $8 \mathrm{~b}$ shows the heat transfer coefficient distribution on the anode surface. The maximum of heat transfer coefficient is about $1.6 \times 10^{4} \mathrm{~W} /\left(\mathrm{m}^{2} \mathrm{~K}\right)$ at the location of the arc attachment. This value is comparable to the values set by $[13,15]$ as a boundary condition.

\section{Conclusions}

In order to predict the electrical and heat transfer properties at the surface of the electrodes (especially at the anode), the $3 \mathrm{D}$, time-dependent, and non-equilibrium plasma torch simulations were performed. In addition to the plasma passage area, electrodes were also inserted into the simulation area. An overview of the general properties of the simulated plasma shows that the model works well. When incorporating the anode into the simulation area, there is no longer any need to apply unrealistic boundary conditions in the electrodeplasma interfaces. The path of electric current passage between the electrodes and the plasma is determined and it is in good agreement with the experimental results. Due to the geometry of the device, the largest amount of electric current passes through the tapered section of the anode. Overlap of the predicted electric current density distribution with the eroded anode sample confirms this. The temperature distribution of the cathode surface varies from $500 \mathrm{~K}$ at the inlet to $3597 \mathrm{~K}$ at the cathode tip which is close to the tungsten melting point $(3643 \mathrm{~K})$. Since the location of the arc attachment is not exactly at the center of the cathode tip, the cathode temperature distribution is not completely Gaussian. Also, the anode surface temperature distribution was predicted. The surface temperature of the anode at the location of the arc attachment is $960 \mathrm{~K}$. The heat transfer coefficient distribution was determined at the anode surface and the obtained values were in agreement with those predicted in the literature.

\section{References}

[1] J. P. Trelles, J. Phys. D. Appl. Phys. 46, 255201 (2013).

[2] M. Baeva, D. Uhrlandt, Plasma Sources Sci. Technol. 20, 035008 (2011). 
[3] Z. Guo, S. Yin, H. Liao, S. Gu, Int. J. Heat Mass Transf. 80, 717 (2015).

[4] Z. Guo, S. Yin, Z. Qian, H. Liao, S. Gu, Comput. Fluids 114, 163 (2015).

[5] J.P. Trelles, C. Chazelas, A. Vardelle, J.V.R. Heberlein, J. Therm. Spray Technol. 18, 728 (2009).

[6] M. Alaya, C. Chazelas, G. Mariaux, A. Vardelle, J. Therm. Spray Technol. 24, 3 (2014).

[7] C. Chazelas, J.P. Trelles, A. Vardelle, J. Therm. Spray Technol. 26, 3 (2017).

[8] J.P. Trelles, J.V.R. Heberlein, E. Pfender, J. Phys. D. Appl. Phys. 40, 5937 (2007).

[9] M. Baeva, R. Kozakov, S. Gorchakov, D. Uhrlandt, Plasma Sources Sci. Technol. 21, 055027 (2012).
[10] ESI Group, CFD-ACE + ver. 2014.0: Modules - Manual.

[11] H.K. Versteeg, W. Malaskekera, An Introduction to Computational Fluid Dynamics: The Finite Volume Method, Pearson Education, 1995.

[12] C.L. Felipini, M.M. Pimenta, J. Phys. Conf. Ser. 591, 012038 (2015).

[13] R. Huang, H. Fukanuma, Y. Uesugi, Y. Tanaka, IEEE Trans. Plasma Sci. 39, 1974 (2011).

[14] J. Miao, D. Yu, X. Cao, Y. Xiang, M. Xiao, J. Yao, Plasma Chem. Plasma Process. 35, 879 (2015).

[15] E. Moreau, C. Chazelas, G. Mariaux, A. Vardelle, J. Therm. Spray Technol. 15, 524 (2006). 